\title{
Richard Labunski, James Madison and the Struggle for the Bill of Rights
}

\section{Philippe Tessier}

\section{Q OpenEdition}

1 Journals

Édition électronique

URL : https://journals.openedition.org/ahrf/10757

DOI : $10.4000 /$ ahrf. 10757

ISSN : 1952-403X

\section{Éditeur :}

Armand Colin, Société des études robespierristes

\section{Édition imprimée}

Date de publication : 1 janvier 2009

Pagination : 213-215

ISBN : 978-2-200-92557-4

ISSN : 0003-4436

\section{Référence électronique}

Philippe Tessier, «Richard Labunski, James Madison and the Struggle for the Bill of Rights », Annales historiques de la Révolution française [En ligne], 355 I janvier-mars 2009, mis en ligne le 30 novembre 2009, consulté le 23 avril 2022. URL : http://journals.openedition.org/ahrf/10757 ; DOI : https:// doi.org/10.4000/ahrf.10757

Ce document a été généré automatiquement le 23 avril 2022.

Tous droits réservés 


\title{
Richard Labunski, James Madison and the Struggle for the Bill of Rights
}

\author{
Philippe Tessier
}

\section{RÉFÉRENCE}

Richard Labunski, James Madison and the Struggle for the Bill of Rights, Oxford, Oxford University Press, 2006, 336 p., ISBN 978-0-19-518105-0, 28 \$.

1 C'est la biographie intellectuelle d'un homme politique et d'un juriste à l'influence discrète mais certaine, James Madison, qu'a publiée Richard Labunski, docteur en sciences politiques et licencié en droit, aujourd'hui enseignant à l'Université du Kentucky, qui est également auteur d'un ouvrage sur la Constitution américaine et de deux essais sur le Premier Amendement.

2 Sa biographie de James Madison se concentre sur le rôle central que joua ce dernier, au milieu de sa vie (1751-1836), dans l'adoption de la Constitution américaine et de ses amendements, dont l'ensemble constitue le Bill of Rights (Déclaration des droits). Labunski passe en effet sous silence l'enfance et la formation de James Madison: diplômé de l'Université de Princeton, protégé de Jefferson, Madison participa, en tant que député à l'assemblée de l'État de Virginie, à la séparation des Églises et de l'État en Virginie à la fin des années 1770, puis il fut élu au Congrès de la Confédération (1781-1789). Dès ses premiers mandats, Madison est décrit par ses collègues comme étant d'un naturel timide, possédé par le souci du détail, et finalement un bourreau de travail parlementaire.

3 Toute l'action politique de Madison ira dès lors dans le sens d'un « fédéralisme ", puis d'un républicanisme modéré - Madison fut un des fondateurs du parti républicain première manière - favorable à un gouvernement fédéral puissant, mais également à une garantie des droits fondamentaux des citoyens et des États, reposant entre autres sur un pouvoir judiciaire fort. Son sens du compromis fut un élément déterminant du succès de la Constitution américaine à ses débuts. 
C'est à partir de la Convention de Philadelphie (été 1787), lors de laquelle fut adoptée la Constitution, que R. Labunski commence son récit. Pendant cette assemblée, se dessina une forte opposition à la Constitution telle qu'elle avait été rédigée (et qui était inspirée du Virginia Plan, établissant entre autres la séparation des pouvoirs, lui-même en grande partie rédigé par Madison). De nombreux hommes politiques de premier plan (George Mason et Patrick Henry, de Virginie, Elbridge Gerry, du Massachusetts) reprochaient à la Constitution d'accorder un trop grand rôle au pouvoir exécutif fédéral, et réclamaient des amendements destinés à protéger les libertés les plus essentielles des citoyens et des États. James Madison, au départ silencieux, se rallia à cette demande qui permit l'adoption, dans un climat de grande tension politique, de la nouvelle Constitution par l'État de Virginie en juin 1788, sous la condition que des amendements lui seraient apportés dès que possible.

5 La ratification de la Constitution par la Virginie était cruciale dans la mesure où un refus de ce texte par la Virginie aurait signifié l'impossibilité pour Washington, leader politique et militaire de la Révolution américaine, et citoyen de l'État de Virginie, d'exercer tout mandat dans l'État fédéral. En effet, la Virginie se serait ainsi exclue des États-Unis et aucun ressortissant de l'État de Virginie n'aurait pu se faire élire à une fonction fédérale.

6 De même, l'État de New-York, où se trouvait un des principaux centres économiques et la capitale provisoire des États-Unis (après Philadelphie et avant la création de Washington D.C.), aurait vraisemblablement suivi la Virginie dans son refus, laissant la Constitution américaine mort-née. James Madison obtint cette ratification par son État d'origine, après une lutte acharnée avec le redoutable orateur anti-fédéraliste Patrick Henry.

7 Ensuite, malgré l'hostilité du pouvoir local virginien, qui avait dessiné des circonscriptions destinées à empêcher son élection, Madison réussit à se faire élire au Congrès de New-York (premier Congrès), après une campagne d'hiver acharnée menée dans des conditions éprouvantes, sous la neige (hiver 1789), et avec le soutien des communautés baptistes reconnaissantes du rôle qu'il avait joué dans la promotion de la liberté religieuse. C'est au Congrès de New-York, sur proposition d'une commission dont Madison faisait partie, que fut voté le Bill of Rights, qui garantissait aux citoyens américains les libertés les plus essentielles, et que furent mis en échec ceux qui estimaient qu'une telle déclaration n'était pas urgente et n'aurait nulle efficacité dans la protection des droits des citoyens.

8 Le Bill of Rights devait garantir certaines libertés fondamentales : il est le résultat, après modification, d'un projet rédigé par la commission dont Madison faisait partie, et qui comprenait une protection de la liberté de la presse, de la liberté d'expression, de la liberté de manifestation et de pétition et de la liberté religieuse (ce qui deviendra le $1^{\mathrm{er}}$ Amendement). Le Bill of Rights protégeait également la liberté de porter des armes (aujourd'hui très discutée, mais qui devait constituer une garantie contre l'arbitraire fédéral et une éventuelle revanche du roi d'Angleterre, dans le $2^{\mathrm{e}}$ Amendement). En outre il comprenait la défense faite aux armées de loger chez l'habitant sauf autorisation de la loi ( $3^{\text {e }}$ Amendement); la protection contre des enquêtes et perquisitions déraisonnables sans cause probable (but upon probable cause, $4^{\mathrm{e}}$ Amendement). Le Bill of Rights établissait aussi la garantie d'un jury d'accusation (Grand Jury) en matière pénale, et du respect de procédures légales nécessaires (due process of law), ainsi que le droit de ne pas témoigner contre soi-même, la garantie de ne pas être 
poursuivi deux fois pour le même crime (double jeopardy) et la protection contre l'expropriation sans juste compensation ( $5^{\mathrm{e}}$ Amendement); le droit à un jugement rapide en matière pénale, devant un jury de jugement impartial et le droit à être assisté d'un avocat ( $6^{\mathrm{e}}$ Amendement) ; le droit à un jury en matière civile, la prohibition des châtiments inhabituels et cruels ( $7^{\mathrm{e}}$ et $8^{\mathrm{e}}$ Amendements). Enfin, le Bill of Rights comprenait des clauses interprétatives de la Constitution, qui interdisent sa lecture contre les droits du peuple ( $9^{\mathrm{e}}$ Amendement) et qui réservent au peuple et aux États tous les droits qui ne sont pas attribués par la Constitution à l'État fédéral $\left(10^{\mathrm{e}}\right.$ Amendement).

9 Dans cette discussion, Madison chercha à préserver le rôle du pouvoir fédéral, en s'opposant à un amendement qui lui interdisait de prélever directement des impôts sur les citoyens. Il s'opposa également à une modification de la rédaction du $10^{\mathrm{e}}$ Amendement, attribuant aux États tout pouvoir qui ne serait pas "expressément " (expressly) attribué à l'État fédéral par la Constitution. L'ajout de cet adverbe, proposé par le représentant Tucker, de Caroline du Sud, aurait empêché l'État fédéral de s'attribuer les pouvoirs que lui accorde implicitement la Constitution, et aurait donc rendu beaucoup plus difficile l'augmentation des pouvoirs de l'État fédéral au XX siècle.

10 James Madison fut le $4^{\mathrm{e}}$ président des États-Unis (de 1809 à 1817), après Washington, John Adams et Thomas Jefferson; il mena avec succès la guerre contre la GrandeBretagne (de 1812 à 1815). En outre, il écrivit pratiquement un tiers des Federalist Papers, qui comptent parmi les commentaires les plus importants de la Constitution américaine, et fut un des initiateurs du « compromis de 1790 », qui aboutit à la fixation de la capitale des États-Unis à Washington.

11 Richard Labunski expose de façon très détaillée et remarquablement précise les discussions parlementaires et juridiques; il dépeint avec vivacité les débats les plus importants, dressant le portrait des hommes politiques-clés de cette période, qu'ils soient partisans ou adversaires de la Constitution. Cet ouvrage, bien illustré, est également un récit vivant et très précis de la vie politique de l'époque, qu'il s'agisse des assemblées fiévreuses et surchauffées de Philadelphie (juin 1787) et de New-York (été 1789) ou des campagnes acharnées de Madison au Sénat et à la Chambre des représentants du premier Congrès (1789). Si l'index et les sources sont bien agencés, il manque cependant une bibliographie à la fin de l'ouvrage.

Le livre de Richard Labunski a le mérite de rendre justice à un homme qui joua un rôle décisif, pour lequel il fut peu reconnu, même si de nombreuses biographies (entre autres, celles de Irving Brant, 1961, celle de Robert A. Rutland, 1987, et celle de Robert A. Goldwin, 1997) lui furent consacrées. Il met en évidence le rôle discret mais crucial des juristes dans les phénomènes révolutionnaires américain et français, mais également les différences fondamentales des deux côtés de l'Atlantique. La démocratie américaine à ses débuts se distingue, malgré des luttes politiques aiguës qui finissaient parfois en duels, par une culture du consensus, de l'action politique légale et du fairplay, comme en témoigne le discours de Patrick Henry, le 28 juin 1788, après l'adoption de la Constitution - dont il était un fervent opposant - par l'assemblée de l'État de Virginie: "J'ai fait énergiquement mon devoir en m'opposant à la Constitution aux lieux et places convenables [in the proper place], et avec tous les pouvoirs que je possédais. La question a été pleinement discutée et tranchée, et les vrais et fidèles républicains doivent rentrer chez eux. Ils doivent chérir la nouvelle constitution, et 
respecter avec loyauté les règles du jeu [give it fair play], la soutenir également, afin que le pouvoir fédéral puisse s'exercer librement et sans entraves ».

13 Avec la différence de conditions géographiques que constituait l'existence d'un vaste espace décentralisé, cette culture politique du consensus et de la légalité est peut-être ce qui décida du destin pacifique de la Révolution américaine. Le livre de R. Labunski en est un convaincant témoignage. 